\title{
A Delayed Occurrence of Surfactant Proteins in An Extremely Preterm Infant
}

\author{
Toshihiko Nakamura ${ }^{1 *}$, Yuki Sakai ${ }^{1,2}$, Daisuke Hatanaka ${ }^{1}$, Michiko Kusakari ${ }^{1}$, Hidehiro \\ Takahashi $^{1}$ and Atsushi Saito ${ }^{3}$ \\ ${ }^{1}$ Department of Neonatology, Japanese Red Cross Musashino Hospital, Musashino, Tokyo, Japan \\ ${ }^{2}$ Department of Pediatrics, National Defense Medical College, Tokorozawa, Saitama, Japan \\ ${ }^{3}$ Department of Respiratory Medicine and Allergology, Sapporo Medical University School of Medicine, Sapporo, Japan \\ *Corresponding author: Toshihiko Nakamura, Department of Neonatology, Japanese Red Cross Musashino Hospital, \\ Kyonan-cho 1-26-1, Musashino, Tokyo 180-8610, Japan
}

ARTICLE INFO

Received: 幽 September 07, 2020

Published: 慧 September 14, 2020

Citation: Xian-bin Zhou, Min Quan, Kuai Ma, Li-ping Ye, Xin-li Mao, Jian-fen Wu, Shiwen Xu, Cai yue, Bin-bin Gu, Yu-Zhang, Li Wang, Jin-bang Peng, Chi Liu, Shao-wei Li. A Delayed Occurrence of Surfactant Proteins in An Extremely Preterm Infant. Biomed J Sci \& Tech Res 30(2)-2020. BJSTR. MS.ID.004937.

Keywords: Extremely Preterm Infant; KL6; Repeat Surfactant Therapy; Respiratory Distress Syndrome; Surfactant Proteins

Abbreviations: HFO: High-Frequency Oscillatory, RDS: Respiratory Distress Syndrome, TA: Tracheal Aspirates

\begin{abstract}
We report an infant with significantly delayed pulmonary surfactant protein secretion, whose clinical course is clearly different from that of a newborn infant with congenital alveolar proteinosis due to abnormalities of pulmonary surfactant protein (SP) B and SP-C genes. The case was female infant born weighting $1049 \mathrm{~g}$ at 26 weeks of gestation. After instillation of artificial lung surfactant as neonatal respiratory distress syndrome in the early postnatal period, respiratory management was started with highfrequency oscillation ventilation. Although the respiratory setting was temporarily weaned after that, the respiratory condition worsened from 3 days of age. As a result, it was necessary to instill the artificial lung surfactant 32 times in total by 35 days of age. This case was clinically inconsistent with congenital alveolar proteinosis based on both the fact that there was no increase in tracheal secretions in abnormal surfactant proteins and the concentrations and changes of serum KL-6, SP-A and SP-D. Pulmonary surfactant protein analysis of tracheal aspirate revealed a significantly delayed secretion of SP-C. The patient was discharged at 134 days of age without home oxygen therapy and is now well at age 2 years with normal growth and development. There is no respiratory infection that requires hospitalization. Unlike the usual clinical course such as RDS and congenital pulmonary alveolar proteinosis, this case is the second case suggesting a new specific disease as extreme delayed secretion of SP-C.
\end{abstract}

\section{Case Report}

A 1049-g female infant was born to a 40-year-old G2P1 mother at 26 weeks gestation via emergency cesarean section due to an indication of placental abruption. After birth, the infant underwent immediate tracheal intubation, and the first dose of artificial lung surfactant (Surfacten $®$ ) was instilled. High-Frequency Oscillatory (HFO) ventilation mode was selected as the initial ventilator setting. However, further improvement of respiratory distress syndrome (RDS) was necessary (Figure 1), and two additional doses of artificial lung surfactant were given on the same day. The HFO mode was combined with intermittent mandatory ventilation to stabilize the infant's respiratory condition as a rescue mode. Because she required an $\mathrm{F}_{1} \mathrm{O}_{2}$ of 0.5 from the age of 3 days to maintain an $\mathrm{SpO}_{2}$ above $90 \%$, additional surfactant was required. She required 32 doses of surfactant up to 35 days of age. Thereafter, we were able to gradually reduce the respiratory settings and succeeded in planned extubation at age 50 days.

Oxygen administration was stopped at age 91 days (Figure 2 ), and she was discharged well at age 134 days. Her corrected gestational age was $46 \mathrm{w} 0 \mathrm{~d}$, and she weighed 4842g (Figure 3). Tracheal aspirates (TA) were obtained as follows. After instillation 
of $0.5 \mathrm{~mL}$ saline, the airway was suctioned with a $4 \mathrm{~F}$ catheter attached to a suction trap to recover the aspirates. The suction catheter lumen was then rinsed with saline, of which about $2 \mathrm{~mL}$ in total was added to the trap. All TA were frozen at $-20^{\circ} \mathrm{C}$ until assayed. Tricine/sodium dodecyl sulfate polyacrylamide gel electrophoresis was performed with $20 \mu \mathrm{g}$ of total protein, and immunoblotting was performed for surfactant protein (SP)-B and SP-C as previously described [1]. Commercially available ELISA kits for SP-A and SP-D of TA and serum were used. Data for SP concentrations are expressed as the standard value divided by albumin $(\mu \mathrm{g} / \mathrm{mg}$ albumin). This study was approved by the ethics committee of the Japanese Red Cross Musashino Hospital.

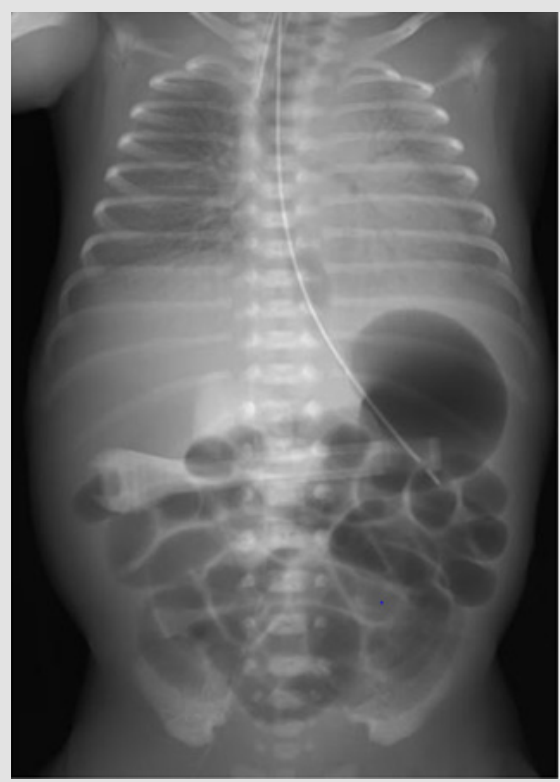

Figure 1: X-ray findings on admission to the NICU after the first administration of artificial lung surfactant. Permeability of both lung fields was poor, the cardiac shadow was unclear, and air bronchogram was also prominent. The classification of Bomsel stage III was consistent with the finding of RDS.

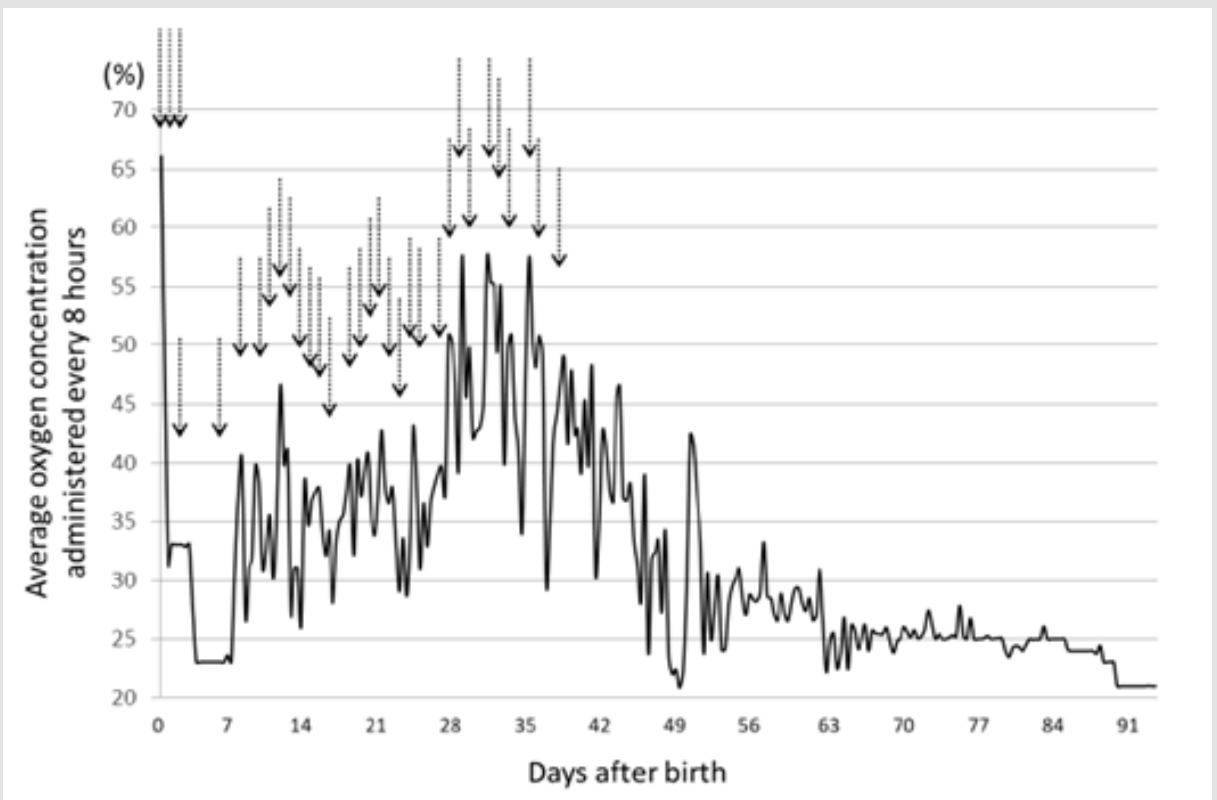

Figure 2: Graph of the change in average $\mathrm{O}_{2}$ concentration every 8 hours and the administration of repeated doses of artificial lung surfactant (indicated by arrows). We administered artificial lung surfactant 3 times at 0 days of age, and lowered the administered $\mathrm{O}_{2}$ concentration once, but only for 1 week. Thereafter, the $\mathrm{O}_{2}$ concentration had to be increased. Surfactant was administered at intervals of about 1 day, and it became possible to begin lower of the administered $\mathrm{O}_{2}$ concentration again from the age of 35 days. The $\mathrm{O}_{2}$ administration was stopped at 91 days. 

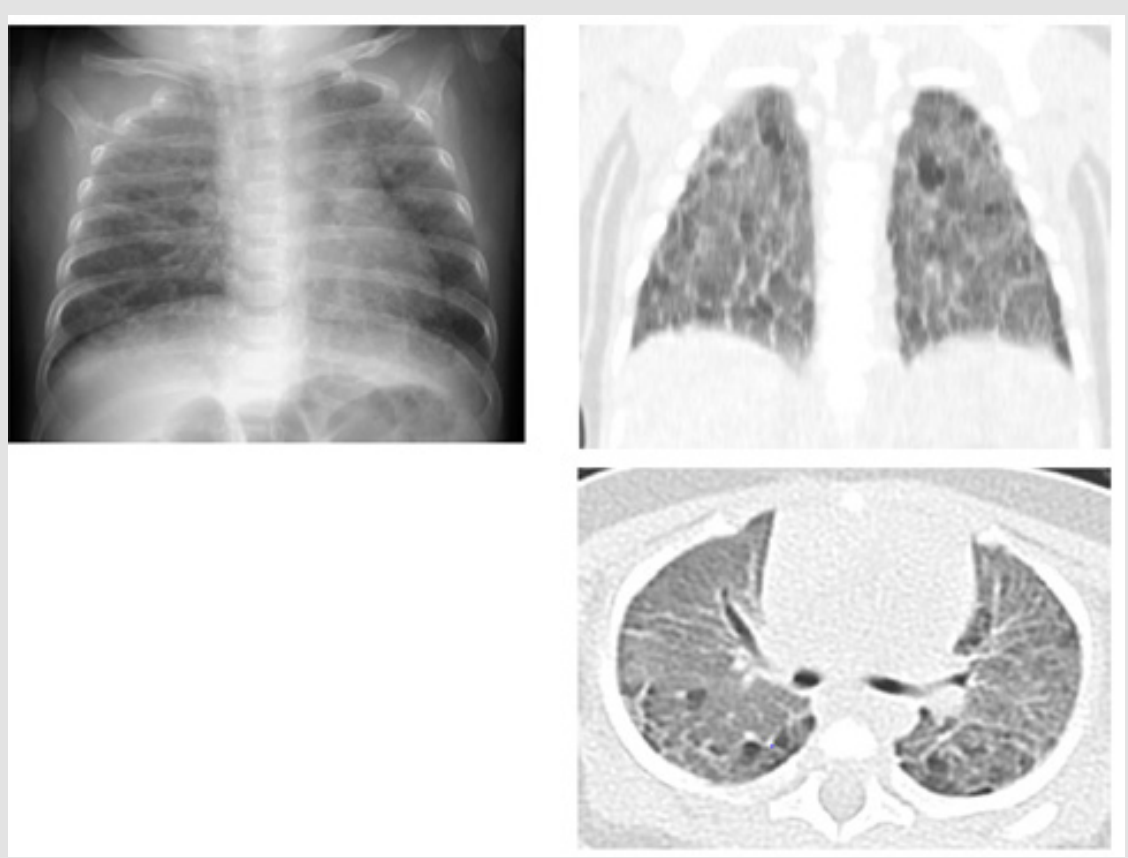

Figure 3: Chest $X$-ray findings and high-resolution CT before discharge on day 105. Although lung permeability was improving, it was still insufficient. The CT images show conspicuous areas of ground-glass opacities accompanied by thickening of the interlobar membranes. A large number of emphysematous cysts were found in the posterior lung fields.

The parents of this infant were informed of the study design, and their written informed consent was obtained for submission of this case report. Western blotting revealed the presence of a considerable amount of SP-B in TA at age 25 days, and the presence of SP-C was confirmed in TA from age 30 days (Figure 4). Serum SP-D increased slightly, peaked at age 30 days, and then decreased. Very little SP-A was detected in blood and TA, but it gradually increased after this age (Figure 5). KL-6 and urinary $\beta 2 \mathrm{MG}$ levels increased up to the age of 30 days and then decreased (Figure 6). We already reported in this journal an extremely preterm infant with no SP gene abnormalities but with a markedly delayed appearance of SP-B and SP-C in TA [2]. The present case is characterized by normal secretion of SP-B and SP-D but insufficient secretion of SP-A and SP-C for about 1 month. Currently, comprehensive gene testing is becoming popular, and urgent genetic testing is recommended for unexplained neonatal respiratory disorders [3].

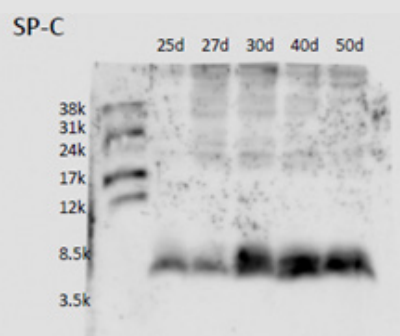

SP-B 25d $27 \mathrm{~d} 30 \mathrm{~d} 40 \mathrm{~d} 50 \mathrm{~d}$

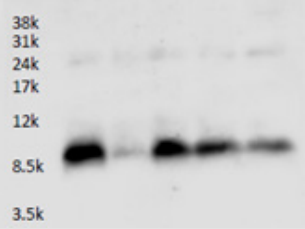

Figure 3: Western blot analysis of surfactant proteins (SP-B and SP-C) in large aggregate surfactant: SDS-PAGE was performed with $20 \mu \mathrm{g}$ of total protein, and immunoblotting was performed for SP-B and SP-C.1 The mature form of SP-B was already found on the $25^{\text {th }}$ day of life, but that of SP-C was not fully expressed until after the 30th day of life. 


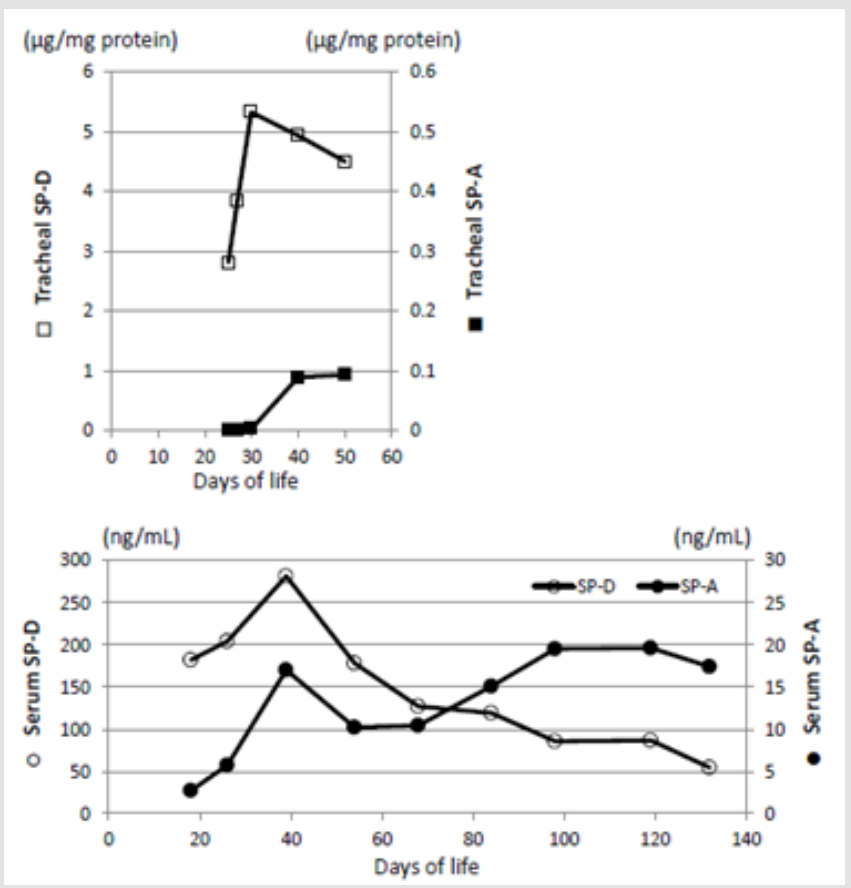

Figure 5: Changes in SP-A and SP-D concentrations in tracheal aspirates and serum. SP-A is present in tracheal aspirate and serum at a level of only about $1 / 10$ that of SP-D. In patients with congenital alveolar proteinosis, serum levels of SP-A and SP-D increase to several 100 or several 1000ng/mL, but this infant's SP-A remained low for a period of one month after birth before increasing. The level was maintained thereafter, and SP-D also increased by a factor of 2-3 to normalize at 3 months of age.

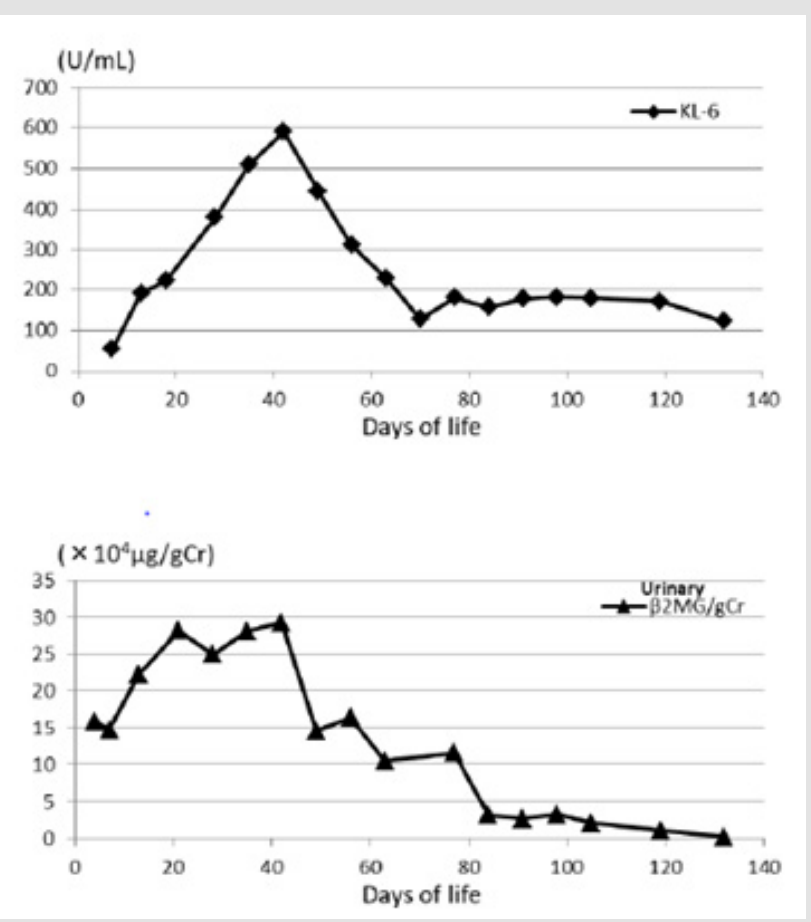

Figure 6: Changes in serum KL-6 and urinary $\beta 2$-microglobulin $(\beta 2 \mathrm{MG})$ concentrations. KL-6 increased in parallel with SP-D and peaked at the age of 40 days. The rise in KL-6 was also about double the reference value. When urinary $\beta 2 M G$ is normalized by the creatinine level, it exceeds $15,000 \mu \mathrm{g} / \mathrm{gCr}$ in infants with chronic lung disease after 2 weeks of age. However, in the present patient, it peaked at about the same time as KL-6 and SP-D, indicating that the hypercytokinemia subsided at around 40 days of age. 
However, Kroner et al. reported that it is difficult to predict the future clinical course even if SP-C deficiency is diagnosed genetically. [4] Congenital alveolar proteinosis caused by SP-B and SP-C deficiency is characterized by elevated serum SP-A, SP-D, and KL-6, and artificial lung surfactant replacement therapy has been shown to have almost no effect. [5] In the present patient, not SP-A, but only serum SP-D and KL-6 mildly increased, gradually decreased, and then remained within the normal range until the day of discharge, indicating that this is not the diagnosis for this patient. When surfactant was administered, FIO2 could be reduced for about 1 day as shown in Figure 2. The infant no longer required repeated doses of artificial lung surfactant from about 1 month after birth, when sufficient SP-C was found in her TA, and at the same time, the administered FIO2 could be reduced. It is possible that the lung tissue damage was minimized by frequent administration of artificial lung surfactant, which may have improved the prognosis. If an extremely preterm infant with a low amount of TA requires frequent doses of surfactant from the early postnatal period, an analysis of surfactant profiles of the TA should be performed immediately.

\section{Disclosure}

The authors declare no conflict of interest.

\section{ISSN: 2574-1241}

DOI: 10.26717/BJSTR.2020.30.004937

Toshihiko Nakamura. Biomed J Sci \& Tech Res

This work is licensed under Creative Commons Attribution 4.0 License

Submission Link: https://biomedres.us/submit-manuscript.php

\section{Author Contributions}

T.N. was responsible for diagnosis and treatment and wrote the manuscript. Y.S., D.H., M.K. and H.T. were responsible for treatment. All authors read and approved the final manuscript.

\section{References}

1 Hamas A, Nogee LM, White FV (2004) Progressive lung disease and surfactant dysfunction with a deletion in surfactant protein $\mathrm{C}$ gene. Am J Respir Cell Mol Biol 30: 771-776.

2 Nakamura T, Shimizu H, Nishitani C, Yoshioka T (2013) Remarkably delayed occurrence of normal surfactant composition in an extremely preterm infant. Pediatr. Int 55: 790-792.

3 Cho K, Yamada M, Agematsu K, Naomichi Matsumoto, Tadashi Ariga, et al. (2018) Heterozygous mutation in OAS1 cause infantile-onset pulmonary alveolar proteinosis with hypogammaglobulinemia. Am J Hum Genet 102(3): 480-486.

4 Kroner C, Reu S, Teusch V, Peter Lohse, Matthias Griese, et al. (2015) Genotype alone does not predict the clinical course of SFTPC deficiency in paediatric patients. Eur Respir J 46(1): 197-206.

5 Al Salmi QA, Walter JN, Colasurdo GN, Hiroki Takahashi, Leland L Fan, et al. (2005) Serum KL-6 and surfactant proteins A and D in pediatric interstitial lung disease. Chest 127(1): 403-407.

$\begin{array}{ll}\text { BIOMEDICAL } & \text { Assets of Publishing with us } \\ \text { RESEARCHES } & \text { - Global archiving of articles } \\ \text { - Immediate, unrestricted online access } & \text { - Rigorous Peer Review Process } \\ & \text { - Authors Retain Copyrights }\end{array}$

